\title{
EL RECONOCIMIENTO DE LA COMUNICACIÓN DE SEÑAS COMO DERECHO INCLUSIVO EN EL PERÚ
}

\author{
THE RECOGNITION OF THE COMMUNICATION OF \\ SIGNS AS AN INCLUSIVE RIGHT IN PERU
}

* Abogado por la Pontificia Universidad Católica del Perú. Magister en Derecho, Doctorado en Derecho por la Pontificia Universidad Católica de Argentina. Profesor Investigador de la Universidad Privada San Juan Bautista y profesor de la Facultad de Derecho de la Universidad Nacional Mayor de San Marcos. Contacto: manuel. bermudez@upsjb.edu.pe,mbermudeztapia@gmail.com ORCID: http://orcid.org/0000-0003-1576-9464

** Abogada por la Universidad Femenina del Sagrado Corazón. Con estudios de Maestría en Docencia Universitaria y Gestión Educativa. Estudios de Derecho Internacional, Religioso Comparado y Humanos en la Universidad de Milán, Italia, Universidad de Montreal, Canadá y Pontificia Universidad Católica del Perú (PUCP). Especialización en Derecho Dominio de 8 idiomas incluido el quechua y manejo de la lengua de señas peruanas. Profesor visitante en la Universidad Autónoma de Chiapas, México. Contacto: nuccia. seminario@unach.mx, nuccia_sh@hotmail.com ORCID: https://orcid.org/0000-0002-1805-7780

Manuel Bermúdez Tapia* Nuccia Seminario Hurtado**

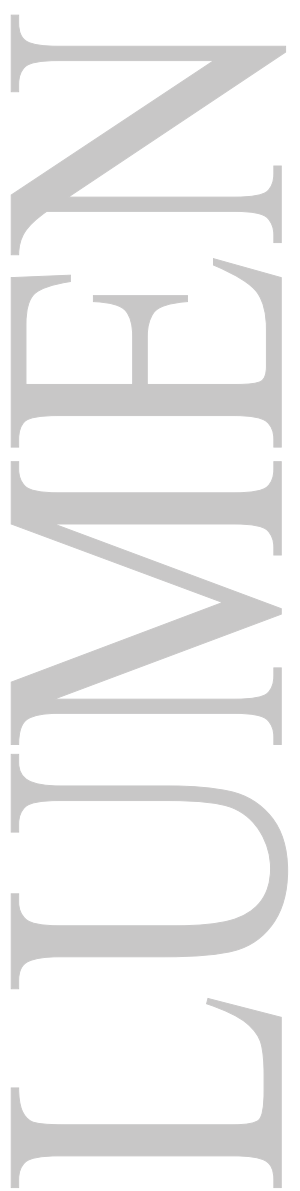




\title{
EL RECONOCIMIENTO DE LA COMUNICACIÓN DE SEÑAS COMO DERECHO INCLUSIVO EN EL PERÚ
}

\author{
THE RECOGNITION OF THE COMMUNICATION OF SIGNS \\ AS AN INCLUSIVE RIGHT IN PERU
}

Manuel Bermúdez Tapia

Nuccia Seminario Hurtado

\begin{abstract}
RESUMEN
El presente artículo plantea evaluar el alcance que genera la comunicación de señas como medio comunicativo legítimo, válido y formal, el cual debe ser reconocido a favor de la comunidad sorda en todo el territorio peruano. En tal sentido, la complementariedad de derechos de naturaleza constitucional aplicables al ámbito judicial, procesal y comunicativo debe ser evaluado de una manera especial y humana, por cuanto la comunidad sorda ha sido atendida en forma equivalente a una persona normal en el trámite de un proceso judicial, generándose una condición limitativa porque en la mayor parte de las Cortes Superiores de Justicia del país no cuentan con intérpretes oficiales en este mecanismo de comunicación.
\end{abstract}

La propuesta de evaluar el alcance de la comunicación como medio de expresión en la participación y accesibilidad a derechos en el ámbito judicial permite detallar que en los casos en los cuales se registre la participación de una persona con limitación auditiva o comunicativa oral, la participación de una persona que le sirva como interprete debe ser considerado como equivalente a lo dispuesto por el inciso $19^{\circ}$ del artículo $2^{\circ}$ de la Constitución y el artículo $8^{\circ}$ de la Convención Americana sobre Derechos Humanos.

\section{PALABRAS CLAVE:}

Derechos Humanos; Derechos Lingüísticos; Debido proceso y Tutela Judicial Efectiva; Accesibilidad a la justicia; Derecho a un intérprete; Socialización del proceso judicial.

\begin{abstract}
This article proposes to evaluate the scope generated by the communication of signs as a legitimate, valid and formal communication medium, which must be recognized in favor of the deaf community throughout the Peruvian territory. In this sense, the complementarity of rights of a constitutional nature applicable to the judicial, procedural and communicative field must be evaluated in a special and humane way, since the deaf community has been attended in an equivalent way to a normal person in the process of a process judicial, generating a limiting condition because in most of the Superior Courts of Justice of the country they do not have official interpreters in this communication mechanism.
\end{abstract}

The proposal to evaluate the scope of communication as a means of expression in the participation and accessibility of rights in the judicial sphere allows to detail that in the cases in which the participation of a person with hearing or oral communication limitations is registered, the participation of a person who serves as an interpreter must be considered equivalent to the provisions of subsection 19 of Article 2 of the Constitution and Article 8 of the American Convention on Human Rights.

\section{KEY WORDS:}

Human rights; Linguistic Rights; Due process and Effective Judicial Protection; Accessibility to justice; Right to an interpreter; Socialization of the judicial process. 


\section{INTRODUCCIÓN}

La evaluación de las condiciones procesales, humanas y sociales que se desarrollan en un trámite judicial requieren de una evaluación especial cuando una persona con alguna condición limitativa se encuentra participando (Bermúdez-Tapia, 2013, p. 45).

La tutela de derechos de grupos vulnerables o en situación de riesgo es un factor que permite detallar la socialización del proceso judicial que garantiza la accesibilidad a la justicia y permite el desarrollo de principios-garantías en el orden procesal y constitucional como la tutela judicial efectiva, el debido proceso, la imparcialidad judicial, la comunicación en el idioma o modalidad personal, entre otros (Bermúdez-Tapia, 2007).

Sin embargo, el reconocimiento de estos derechos no siempre se ha ejecutado de modo diligente o eficiente, en particular porque las condiciones institucionales, logísticas, de atención de apoyo humano especializado o pericial a la actividad judicial no siempre es garantizada en el Poder Judicial, Ministerio Público o el mismo Tribunal Constitucional.

En este sentido, la realidad social, económica y cultural de un país multilingüe, pluricultural y con contrastes diferenciados y de exclusión de poblaciones permite detallar la importancia que genera la humanización del proceso judicial, a través de la actividad de los magistrados, cuando deben evaluar el derecho de una persona que integra una comunidad vulnerable o en situación de riesgo.

Complementariamente esta labor se ve reforzada por la legislación que ha venido implementándose desde haces tres décadas, en particular porque la Convención Americana sobre Derechos Humanos ha logrado generar un hito histórico que se ha complementado con las acciones de la Organización de Naciones Unidas con la promulgación de Convenios, Tratados, Acuerdos o Pactos a favor de mujeres, niños, indígenas personas de tercera edad, migrantes, especialmente.

De este modo, en los últimos años, la evaluación de condiciones y características particulares que limitan la tutela de derechos de determinadas personas permite evaluar la necesaria ejecución de acciones tuitivas y coyunturales que permitirían la equivalencia de condiciones y derechos entre las partes procesales, que en el presente texto se centran en la evaluación de los derechos de las personas sordomudas o con problemas de comunicación.

El desarrollo del presente texto tiene una metodología hermenéutica de tipo cualitativo con un enfoque interdisciplinario y sistemático desarrollado en capítulos estructurados en función a la evaluación de la participación procesal de personas sordomudas en un proceso judicial, siendo el objetivo del trabajo el exponer las limitaciones que tienen estas personas en el desarrollo de un proceso judicial en caso no cuenten con el apoyo de una persona que ejecute la interpretación de su comunicación, de forma oficial y oportuna.

\section{El contexto panorámico de la realidad judicial peruana con respecto de la participación de sordomudos como parte procesal o parte involucrada en un proceso judicial}

El 9 de octubre del 2020, se hizo pública la ejecución de una audiencia en el Sexto Juzgado de Paz Letrado de la Provincia de Cajamarca en la Corte Superior de Justicia de Cajamarca, en la cual una parte procesal planteaba alimentos como pretensión y ambas partes procesales registraban discapacidad auditiva (Andina, 2020).

Un hecho particular y especial por cuanto en este caso, las dos partes registraban inconvenientes a nivel de una misma discapacidad pero que el trámite en sí mismo pudo ser atendido en forma oportuna, diligente y sobre todo a nivel preliminar. 
Referencia muy común en el ámbito de los juzgados de paz, que son los órganos judiciales que cuentan con un sistema de interacción con los justiciables mucho más directo, inmediato y oportuno, con lo cual la sobre carga judicial no constituye un elemento que condiciona el trámite del proceso en la misma referencia que sí produce en los otros órganos judiciales.

Complementariamente, según datos oficiales que reproduce "Perú 21" al 2015, se reportaban 532 mil personas con alguna discapacidad auditiva, cuyas características pueden diferenciarse:

a) Personas que usan un audífono para efectos de manejar su comunicación auditiva de modo más eficiente.

b) Personas que podrían acceder a una operación quirúrgica para mejorar su capacidad auditiva con el uso de algún dispositivo electrónico.

c) Personas cuya capacidad económica o con la opción de comunicarse con lenguaje de señas.

En este ámbito, nótese que estas cifras podrían incrementarse porque no todas las personas con limitaciones auditivas se han registrado en la Federación Nacional de Sordos del Perú o en el CONADIS (CONADIS, 2020) y esto limita la percepción de las acciones que pudiera ejecutar el Estado cuando tenga que comunicarse con estas personas en algún trámite o administrativo o judicial.

Una situación que se agudiza en el ámbito educativo porque en el país sólo existe un colegio especial, a nivel de educación de primaria y en otro de educación secundaria en todo el país (Perú $21,2015)$, con lo cual las referencias nos permiten indicar una situación que no ha sido visibilizada ni atendida debidamente.

Asimismo, la Defensoría del Pueblo en el 2018, señaló que "El Estado ha reconocido oficialmente la lengua de señas peruana y ha dispuesto que todas las instituciones públicas y privadas que brindan atención al público, cuenten con el servicio de intérpretes de esta lengua de manera gratuita, y por ende deben ser atendidas por un médico, presentar una denuncia en la comisaría, sacar una partida de nacimiento, cobrar cheques, participar en un partido político, votar, y, tienen derecho a recibir una educación de calidad y el contenido de los programas informativos de la televisión, ambos en lenguaje de señas" (Defensoría del Pueblo, 2018).

De ello podemos señalar que el Estado Peruano reconoce a la comunidad sorda como sujetos con derechos de forma individual y de manera autónoma como comunidad vulnerable o en situación de riesgo, con lo cual se hace necesario evaluar la participación de estas personas en el ámbito judicial, porque no todas las Cortes Superiores de Justicia en el país disponen de un personal especializado como intérpretes de señas.

Bajo estos alcances, la comunicación de señas debe ser reconocido como un derecho derivado de la capacidad y comunicación lingüística (Bermúdez-Tapia, 2009), sobre la cual se le debe reconocer la opción de actuar con un intérprete a toda persona que lo requiera, porque está vinculado con una esfera que garantiza la progresividad de los Derechos Humanos.

\section{La comunicación con señas como un derecho derivado del derecho a comunicarse diligentemente}

La comunicación es parte de la realización del pleno desarrollo de la persona humana, es ese sentido, se debe entender al aspecto comunicativo como un factor intrínseco en el aspecto de la socialización y la familiarización, y un proceso interactivo e interpersonal compuesto por elementos que interactúan constantemente. 
En el ámbito político permite la ejecución de derechos de modo directo, autónomo y diligente, con lo cual es posible identificar una serie de condiciones que pueden derivar en situaciones negativas si es que por medio de la incapacidad de comunicarse no se puede acceder a servicios públicos (Bermúdez-Tapia, 2001, p. 333), que, en este ámbito del texto, están limitados al contexto de la accesibilidad a servicios de justicia.

Es decir, la comunicación es entendida como el intercambio y negociación de información (conceptual, sociocultural, afectiva) entre al menos dos individuos por medio del uso de símbolos verbales y no verbales, de modos orales y escritos/visuales y de procesos de producción y comprensión" (Canales, 1983).

La comunicación, como acto humano forma parte del proceso biosocial del ser humano sobre la cual está en un estado natural que supera para organizarse en un contexto político para así generar y legitimar al Estado, como detallaba Hobbes (Ávila et al, 2016, p. 149).

Como elemento comunicativo, el humano emplea las funciones de la memoria, tanto neurológicas como psicológicas, las cuales se producen en el sistema nervioso central porque ello le garantiza la posibilidad de mantener un único sistema de comunicación con sus semejantes. Aquí, un estímulo externo produce una señal o registro, que se guardará en la memoria, para ser utilizada cuando el hablante encuentre la ocasión para ello y demostrar así la capacidad que tiene el ser humano de seleccionar este recurso para usarlo arbitrariamente (distinción con respecto a la comunicación simple y primitiva del animal) (Guardia, 2009, p.15)

Referencias que permiten detallar que la comunidad sorda cuenta la garantía para emplear la comunicación con señas o emplear sistemas lingüísticos de carácter visual, espacial, gestual y manual cuando quiera interactuar con terceras personas más aún cuando se debaten sus derechos y obligaciones en el ámbito judicial, conforme a la Ley $N^{\circ} 29535$, Ley que Otorga Reconocimiento Oficial a la Lengua de Señas Peruana y el Decreto Supremo N006-2017-MIMP, que aprueba el Reglamento de la Ley $N^{\circ}$ 29535, Ley que Otorga Reconocimiento Oficial a la Lengua de Señas Peruana.

Concerniente a su reconocimiento desde la perspectiva del sistema de protección universal y regional de los derechos humanos, así como también desde la perspectiva constitucional, podemos fundamentar esta posición en función a la complementariedad, de:

a) Pacto de Derecho Civiles y Políticos (1976) en su artículo 27 que señala que "en los Estados en que existan minorías étnicas, religiosas o lingüísticas, no se negará a las personas que pertenezcan a dichas minorías el derecho que les corresponde, en común con los demás miembros de su grupo, a tener su propia vida cultural, a profesar y practicar su propia religión y a emplear su propio idioma".

b) Pacto Internacional de Derechos Económicos, Sociales y Culturales (1976), en su artículo "1.1 Todos los pueblos tienen el derecho de libre determinación. En virtud de este derecho establecen libremente su condición política y proveen asimismo a su desarrollo económico, social y cultural".

c) La Convención Americana de Derechos Humanos (1969), en su artículo 24 protege la igualdad ante la ley señalando que "Todas las personas son iguales ante la ley. En consecuencia, tienen derecho, sin discriminación, a igual protección de la ley".

d) Convención sobre persona con discapacidad (2007) en su artículo 2, señala todo lo concerniente a los estándares educativos, enfatizando que "la «comunicación» incluirá los 
lenguajes, la visualización de textos, el Braille, la comunicación táctil, los macrotipos, los dispositivos multimedia de fácil acceso, así como el lenguaje escrito, los sistemas auditivos, el lenguaje sencillo, los medios de voz digitalizada y otros modos, medios y formatos aumentativos 0 alternativos de comunicación, incluida la tecnología de la información y las comunicaciones de fácil acceso; Por «lenguaje» se entenderá tanto el lenguaje oral como la lengua de señas y otras formas de comunicación no verbal;

e) El artículo $48^{\circ}$ de la Constitución Política del Perú que regula los derechos lingüísticos en el país, tanto de modo individual, colectivo como difuso, por cuanto no puede ejecutarse una evaluación excluyente de condiciones en función a la evaluación extensiva de los derechos naturales que permiten ejecutar una evaluación en el Bloque de Constitucionalidad en el Perú.

f) Ley $N^{\circ} 29535$. Ley que otorga reconocimiento oficial a la lengua de señas peruanas, en su artículo 1 objeto denota que se debe otorgar reconocimiento oficial y regular la lengua de señas peruana como lengua de las personas con discapacidad auditiva en todo el territorio nacional.

g) Decreto Supremo № 006-2017-MIMP, que aprueba el Reglamento de la Ley № 29535, Ley que Otorga Reconocimiento Oficial a la Lengua de Señas Peruana, como dispositivo legal complementario de la norma antes citada.

\section{La tutela de derechos en comunidades vulnerables en el ámbito comunicativo}

Entendemos por "vulnerable" a la población que no puede atender sus necesidades por sus propios medios, por lo cual necesita ayuda externa para salir adelante. Quienes están en posibilidad de atender a los grupos vulnerables son a nuestro entender, por una parte, los profesionales en las disciplinas sociales y por otra el Estado a través de las políticas sociales. (Vega, s/f. p.10)

En ese contexto, consideramos que son considerados como grupos vulnerables, todas las minorías lingüísticas, culturales, religiosos y políticas, que necesitan que sus derechos individuales y colectivos se les proteja debidamente en un contexto social-multicultural, porque asumen una condición limitativa en función a elementos históricos y de exclusión social, económica e inclusive política en el país (Poder Judicial, 2012).

En ese sentido, la comunidad sorda forma parte de la referencia a la cual se le debe considerar como grupo vulnerable porque representan un colectivo significativamente limitado frente a la población nacional, de forma equivalente a las minorías de características sociales, culturales, lingüísticas o étnicas (OHCHR, 2020).

Sin embargo, la comunidad sorda no registra un elemento de visibilidad como la que registran otras comunidades vulnerables, por ello es importante especificar que al evidenciarse contextos de discriminación, de exclusión o de generación de condiciones negativas, las personas con sordera pueden verse afectados en la misma medida en la cual se les afecta a las personas de otros grupos vulnerables, siendo importante definir las políticas de integración social para así reducir las brechas de exclusión (Pérez, 2014, p. 272).

La pertenencia a la comunidad sorda, detalla García Fernández, se define por las siguientes condiciones:

a) Por el uso de la comunicación que emplea signos. 
b) Los sentimientos de identidad grupal, en particular porque la comunicación con señas los identifica como parte de un colectivo.

c) El autoreconocimiento y la identificación como sordo, el cual genera una condición social que se ha generado en función a la alta exclusión social que se registra en nuestro país, en particular porque las referencias sociales negativas han promovido la ejecución de matrimonios endogámicos.

En este sentido, el $83 \%$ de las personas sordas reportan tener una pareja también sorda, mientras un 16,5\% tienen pareja sin problemas auditivos (García, 2004, 39-40).

Kyle y Woll detallaban que la identidad sorda hace énfasis en los sentimientos de grupo de los sordos y en la lengua de señas como medio efectivo de comunicación. Al saber los sordos que disponen de una comunicación efectiva marcan una diferencia con los demás integrantes de la comunidad (que son oyentes) y permite la generación de una condición subjetiva porque desaparece el imaginario de una autopercepción de ser individuos discapacitados (1985, p. 47).

En ese sentido denotamos que la comunicación de señas es un medio comunicativo, "que va tomando forma de país a país produciéndose así los diferentes lenguajes de señas en cada uno de los mismos. Tenemos, por ejemplo, al Lenguaje de Señas Peruanas o LSP, el Lenguaje de Señas Americanas (American Sign Language). ASL EI ISN o Lengua de Señas de Nicaragua, el cual posteriormente se ha denominado Idioma de Señas como un sistema de comunicación gobernado por reglas, el cual asegura la comunicación entre los usuarios de una comunidad." (García, 2002, p. 38).

\section{La comunidad sorda en el Perú}

De los usos registrados en el Diccionario de la lengua española (DLE), se entiende como comunidad a un conjunto de personas vinculadas por características o intereses comunes. Así se constituye la Comunidad Sorda como aquel grupo de personas unidas por tener una comunicación de señas que los identifica y genera una condición única al sufrir de una condición limitativa en sus capacidades, la sordera. Sin embargo, esta comunidad no está conformada solo por sordos, sino también por oyentes vinculados a aquellos por relaciones familiares y amicales (Cuti, 2018, p. 29)

Es así que la comunidad sorda es considerada como un grupo colectivo que posee usos, costumbres y hábitos propios, entre ellos el uso de la lengua de señas como medio comunicativo, a fin de utilizar un lenguaje que incluye gestos corporales para expresar su opinión y otros.

Respecto del contexto normativo, es necesario indicar que en 1996 el Ministerio de Educación publicó un manual sobre la comunicación de señas del país (Min. Educación, 1996). Dicha publicación fue continuada con un segundo tomo en 1997 (Min. Educación, 1996).

Para el año 2002 aparecen dos estudios de Irene García Benavides, en los que se aborda la existencia de la Comunicación de Señas (CdS) desde un punto de vista lingüístico. La autora argumenta a favor de considerar la CdS como una lengua natural, para lo cual describe la estructura interna de algunas señas de la CdS y ofrece ejemplos del funcionamiento de su sintaxis (Oviedo, 2015). 


\section{La comunicación con señas peruanas como derecho a favor de la comunidad sorda en el Perú}

La Ley $\mathrm{N}^{\circ} 29535$, Ley que otorga reconocimiento oficial a la lengua de señas peruana (CdS) en su artículo 3 señala que "el Estado promueve las actividades de investigación, enseñanza y difusión de la lengua de señas peruana y otros sistemas de comunicación alternativos validados por el Ministerio de Educación, para efectos de facilitar el acceso de las personas con discapacidad auditiva a los servicios públicos y el ejercicio de los derechos y libertades constitucionales".

De este modo, se regulaba el reconocimiento del acceso a la información como derecho fundamental intrínseco del grupo que posee una discapacidad auditiva, por ello, algunas entidades estatales han establecido directivas a fin de garantizar la vigencia efectiva de este derecho:

a) La Defensoría del Pueblo implementó el servicio de intérprete de lengua de señas en su oficina de Tacna, para que las personas con discapacidad auditiva en esta región puedan alertar adecuada y oportunamente (Defensoría del Pueblo, 2019).

b) En cumplimiento de la Ley $\mathrm{N}^{\circ} 29535$, que otorga reconocimiento oficial a la lengua de señas, y su Reglamento, aprobado mediante Decreto Supremo N 006-2017- MMP, la Autoridad Nacional del Servicio Civil- SERVIR brinda el servicio de atención y orientación a usuarios con discapacidad auditiva, a través de un intérprete de la lengua de señas peruana (Servir, 2020).

c) En cumplimiento de la Ley $\mathrm{N}^{\circ} 29535$, que otorga reconocimiento oficial a la lengua de señas y su Reglamento, aprobado mediante Decreto Supremo N 006-2017- MMP, el Organismo de Evaluación y Fiscalización Ambiental brinda el servicio de atención y orientación a usuarios con discapacidad auditiva a través de un intérprete de la lengua de señas peruana en la Sede Central y en las Oficinas Desconcentradas y de Enlace.

De esta manera, los usuarios con discapacidad auditiva que requieran efectuar algún trámite o consulta ante el OEFA, pueden realizar la solicitud del servicio de intérprete de la lengua de señas. El servicio podrá ser otorgado, de acuerdo a la necesidad del ciudadano: en el mismo día de solicitado o previa coordinación de una cita con el intérprete (OEFA, 2020).

d) El Ministerio de Defensa accede al servicio de atención especializada para personas con discapacidad auditiva a través de la participación de un intérprete de lengua de señas peruana, para garantizar la accesibilidad de los servicios y la información que se requiera (MINDEF, 2020).

\section{Políticas públicas educativas en el ámbito de la comunicación}

La educación es un derecho fundamental intrínseco y un medio indispensable para la plena realización de otros derechos fundamentales, y permite al ciudadano participar plenamente en la vida social y política en sus comunidades.

En este punto, el Tribunal Constitucional en su jurisprudencia, expediente $\mathrm{N}^{\circ}$ 0091-2005- PA/TC en su fundamento jurídico 6 " (...) ha detallado que este derecho que se garantiza la formación de la persona en libertad y con amplitud de pensamiento, para gozar de una existencia humana plena, es decir, con posibilidades ciertas de desarrollo de las cualidades personales y de participación directa en la vida social" (TC, 2005). 
De ello, debemos señalar que la comunidad sorda, como grupo o minoría cultural y lingüística es acreedor de derechos exclusivos, tales como recibir una educación en su lengua materna. La lengua de señas, como idioma oficial de la comunidad sorda se imparte mediante una educación bilingüe que aborda un modelo lingüístico donde la persona sorda "se vincula e interactúa con la comunidad educativa de forma proficiente, a fin de facilitar el aprendizaje de la lengua de señas peruana y el acercamiento a la cultura sorda de las y los estudiantes con discapacidad auditiva o estudiantes sordos (Reglamento de la Ley 29535, art. 3.7). Es decir, es un usuario nativo o cuasi nativo que coopera con el docente en la educación de niños y niñas sordas, y en su integración a la comunidad sorda peruana." (Ministerio de Educación, 2020).

Es decir, la educación de la comunidad sorda abarca dos perspectivas:

a) Permite el desarrollo de la enseñanza de la lengua de señas, entendida como Comunicación de Señas y un enfoque de igualdad y calidad, a fin de que reciban una educación altamente calificada, tal como indica la Ley General de Educación, Ley $N^{\circ} 28044$, que en su artículo 8 inciso f). detalla: La interculturalidad es un principio que asume como riqueza la diversidad cultural, étnica y lingüística del país.

b) Permite desarrollar objetivos a nivel de país como Políticas de Estado, tal como se detalla en el artículo 9. b), se detalla que uno de los fines de la educación es contribuir a formar una sociedad democrática, solidaria, justa, inclusiva, próspera y tolerante que afirme la identidad nacional sustentada en la diversidad cultural, étnica y lingüística. Complementariamente en el artículo 10, se detalla que se necesita lograr la universalización, calidad y equidad en la educación, se adopta un enfoque intercultural.

Es importante recalcar que, en el Perú, existen más de 5 mil estudiantes con discapacidad auditiva que son atendidos en las modalidades del sistema educativo, en instituciones públicas y privadas a nivel nacional, que requieren una atención humana, técnica y profesional especial y por ello serán beneficiados por la Ley $N^{\circ} 29535$, que reconoce oficialmente a la lengua de señas peruana y cuyo reglamento fue aprobado recientemente.

Además, las instituciones educativas públicas y privadas de los niveles de educación secundaria de las modalidades de Educación Básica Regular, Educación Básica Alternativa y Técnica Productivo y de Educación Superior garantizarán la intervención progresiva de intérpretes para personas con discapacidad auditiva cuando estas lo soliciten y mientras dure su permanencia en dichas instituciones educativas. (Ministerio de Educación, 2017).

\section{Acciones en el ámbito de la implementación de políticas lingüísticas y educativas especiales para la comunidad de sordos}

Se debe garantizar la vigencia efectiva del derecho a comunicarse en la lengua de señas a la comunidad sorda en el Perú, implementando como Política de Estado, mayores estrategias y políticas lingüísticas debidamente capacitadas, a fin de fomentar el progreso de este, en particular en la población que no sufre ninguna discapacidad para así generar un proceso de inclusión mucho más eficiente.

Se debe fomentar el uso de la lengua de señas peruanas en el ámbito social, político, educativo y familiar, como también se debería tener un programa institucional en el Ministerio de educación para que así las lenguas originarias puedan adaptar este mecanismo de comunicación, con lo cual la inclusión social, étnica y lingüística podría garantizar un país mucho más sensible a las características multilingüísticas y pluriculturales del país (Bermúdez-Tapia, 2001). 
Se debe impulsar la participación ciudadana a través de programas de vecindad que refuercen el uso de la lengua de señas peruanas en aquellos determinados espacios geográficos donde existen gran cantidad de miembros quienes hacen parten de la comunidad sorda.

Se debe fortalecer las políticas educativas abocadas en el uso de la lengua de señas, a fin de que se garantice la vigencia efectiva del derecho a la educación en la lengua materna a la comunidad sorda.

Se debe fomentar el estudio de la lengua de señas peruanas a la comunidad oyente, para que de esa manera se formen mayores intérpretes de lengua de señas, a fin de establecer un diálogo socio-cultural y lingüístico.

\section{CONCLUSIONES}

La comunidad sorda, es un grupo colectivo que posee usos, costumbres, hábitos y fomenta el uso de la lengua de señas como medio comunicativo, por ello se reconoce que poseen derechos individuales y colectivos fundamentales intrínsecos para su desarrollo como personas humanas y grupo.

La lengua de señas peruanas está reconocida por la normatividad interna y tiene como objeto garantizar la vigencia efectiva de la lengua de señas como medio comunicativo de la comunidad sorda.

Existen distintos instrumentos jurídicos nacionales e internacionales que protegen el derecho humano lingüísticos de los miembros, quienes conforman la comunidad sorda, desde los culturales y lingüísticos.

El derecho a la educación bilingüe es un derecho fundamental de orden constitucional innato de la comunidad sorda, pues, enmarca la protección lingüística arraigada a la educación.

\section{REFERENCIAS}

- Andina (2020, 9 de octubre) Cajamarca: jueza utiliza intérprete en lengua de señas en juicio por alimentos. https://andina.pe/agencia/noticia-cajamarca-jueza-utiliza-interprete-lenguasenas-juicio-alimentos-817133.aspx

- Ávila Martínez, A., Castellanos Rodríguez, N., Triana Agudelo, A. (2016) La teoría política de Thomas Hobbes y su influencia en la construcción del principio de legalidad en el Estado Moderno. Revista Via luris. (20), 149-162

- Bermúdez-Tapia, M. (2001) Los derechos lingüísticos. Lima: Ediciones Legales

- Bermúdez-Tapia, M. (2001) Categorías de ciudadanía en el Perú, por el goce de derechos fundamentales. Boletín del Instituto Riva Agüero. (28), 333-343

- Bermúdez-Tapia, M. (2007) La constitución a través de las sentencias del Tribunal Constitucional. Lima: Ediciones Legales

- Bermúdez-Tapia, M. (2009) Acceso a la justicia a través del lenguaje y comunicación forense. Los derechos lingüísticos como derecho fundamental ante el Sistema Judicial, en Córdova Shaefer, J. (Ed.) Derechos fundamentales en su jurisprudencia. Lima: Ediciones Caballero Bustamante

- Bermúdez-Tapia, M. (2013) Acceso a la justicia a través del lenguaje y comunicación forense, en Asociación Peruana de Derecho Constitucional (Ed.) Derecho Constitucional. Lima: ARA

- CONADIS (2020) Federación Nacional de Sordos del Perú y CONADIS establecen acuerdos. https://www.conadisperu.gob.pe/notas-informativas/federacion-nacional-desordos-delperu-y-conadis-establecen-acuerdos 
- Decreto Supremo N006-2017-MIMP. Decreto Supremo que aprueba el Reglamento de la Ley $N^{\circ} 29535$, Ley que Otorga Reconocimiento Oficial a la Lengua de Señas Peruana

- Decreto Supremo N006-2017-MIMP. Decreto Supremo que aprueba el Reglamento de la Ley $N^{\circ} 29535$, Ley que Otorga Reconocimiento Oficial a la Lengua de Señas Peruana Defensoría del Pueblo (2018, 30 de enero). "Derechos de las personas sordas". https://www.defensoria. gob.pe/blog/derecho-de-las-personas-sordas-comparte-tus- derechos/

- Defensoría del Pueblo. (2019). Defensoría implementa servicio de lengua de señas para atender a personas sordas en Tacna. https://www.defensoria.gob.pe/defensoria- implementaservicio-de-lengua-de-senas-para-atender-a-personas-sordas-en-tacna/

- García, B. (2004). Cultura, educación e inserción laboral de la comunidad sorda. (tesis doctoral). Granada: Universidad de Granada.

- García, I. (2002). Lenguaje de señas entre niños sordos de padres sordos y oyentes. (tesis de licenciatura). Lima: Universidad Nacional Mayor de San Marcos.

- Guardia, K. (2009). Lenguaje y comunicación. 1ª. ed. - San José_Colección Pedagógica Formación Inicial de Docentes Centroamericanos de Educación Básica. Recuperado de: https://ceccsica.info/sites/default/files/content/Volumen_25.pdf

- Kyle, J. y Woll, B. (1985). Sign language: The study of deaf people and their language. Cambridge: Cambridge University Press.

- Ley $N^{\circ}$ 29535, Ley que Otorga Reconocimiento Oficial a la Lengua de Señas Peruana https:// busquedas.elperuano.pe/normaslegales/ley-que-otorga-reconocimiento-oficial-a-la- lenguade-senas-p-ley-n-29535-496850-3/

- MINDEF (2020, 10 de octubre) Recibir atención mediante un intérprete de lengua de señas peruana para trámites y servicios del MINDEF. https://www.gob.pe/820-recibir-atencionmediante-un-interprete-de-lengua-de-senas-peruana-para-tramites-y-servicios-del-mindef

- Ministerio de Educación (2020, 10 de octubre) Perfil del Modelo Lingüístico. http://www. minedu.gob.pe/modelo-linguistico/pdf/modelo-linguistico.pdf

- Ministerio de Educación. (2017). "Sistema educativo atiende a cinco mil estudiantes con discapacidad auditiva". Recuperado de: http://www.minedu.gob.pe/n/noticia. $p h p ? i d=44025$

- OEFA (2020, 10 de octubre) Servicio de intérprete de la lengua de señas peruana. https:// www.oefa.gob.pe/atencion/discapacidad-auditiva/

- $\mathrm{OHCHR}$ (2020,10 de octubre) Derechos de las minorías. https://www.ohchr.org/documents/ publications/minorityrights_sp.pdf

- Oviedo, A. (2015) Perú, atlas sordo. https://cultura-sorda.org/peru-atlas-sordo/

- Perú 21 (2015, 3 de diciembre) En Perú hay 532,000 personas sordas y solo 23 intérpretes. https://peru21. pe/lima/peru-hay-532-000-personas-sordas-23-interpretes-video-199711noticia/

- Poder Judicial (2012) Plan nacional de acceso a la justicia de personas vulnerables. https:// apps.pj.gob.pe/justiciaentucomunidad/

- Servir (2020, 10 de octubre). Servicio de intérprete de la lengua de señas peruana. https:// www.servir.gob.pe/servicio-de-interprete-de-la-lengua-de-senas-peruana/

- Tribunal Constitucional (2005) Expediente № 0091-2005-PA/TC. https://tc.gob.pe/ jurisprudencia/2006/00091-2005-AA.html

Fecha de recepción: 23 de octubre de 2020

Fecha de aceptación:06 de noviembre de 2020 